\title{
Results of a multi-level therapeutic approach for Alzheimer's disease subjects in the "real world" (CRONOS project): a 36-week follow-up study
}

\author{
Giuseppe Bellelli ${ }^{1}$, Elena Lucchi ${ }^{1}$, Nadia Minicuci ${ }^{2}$, Luca Rozzini ${ }^{3}$, Angelo Bianchetti ${ }^{4}$, \\ Alessandro Padovani ${ }^{3}$, and Marco Trabucchi ${ }^{5}$ \\ ${ }^{1}$ Ancelle della Carità Hospital, Cremona, ${ }^{2}$ Padova Aging Unit, Institute of Neuroscience, National Research \\ Council, ${ }^{3}$ Department of Neurology, University of Brescia, ${ }^{4}$ S. Anna Hospital, Brescia, ${ }^{5}$ Tor Vergata \\ University, Roma, and Geriatric Research Group, Brescia, Italy
}

ABSTRACT. Background and aims: Recently, the Italian Ministry of Health started a national project (CRONOS project), aiming at assessing how a multi-level therapeutic approach - including 2-year free-ofcharge treatment with cholinesterase inhibitors (ChEI), pharmacologic and non-pharmacologic management of behavioral disorders, periodic multi-dimensional assessment, and informal caregivers' counseling - performs in subjects with mild-to-moderate Alzheimer's disease (AD). Five hundred and three Alzheimer Evaluation Units (AEUs) were instituted for this purpose all over Italy. In this paper we present the results of this approach in a large population of $A D$ subjects followed for 36 weeks by 14 AEUs in Eastern Lombardy, Italy. Methods: The project lasted for two years (September 2000-September 2002). Subjects eligible for the CRONOS project had a diagnosis of probable AD, a Mini Mental State Examination (MMSE) score at baseline ranging from 10 to 26, and onset of cognitive disorders between 40 and 90 years of age. Periodic clinical and multi-dimensional assessments, including MMSE, Activities of Daily Living (ADL) and Instrumental Activities of Daily Living (IADL) were made at 12 and 36 weeks; ChE-I doses, psychotropic and antidepressant drugs were also re-assessed at all clinical examinations. Caregivers were instructed about dementia and drug-related problems. Results: Of the 808 subjects who completed the 36week follow-up, 441 were naïves (i.e., never previously treated with ChE-I drugs) and 367 non-naives. At 12 weeks, both naïves (mean variation from baseline $=$ 0.8 points) and non-naïves (mean variation from base- line $=0.5$ points) improved their MMSE scores, while at 36 weeks only naïves improved (mean variation from baseline $=0.1$ ) and non-naïves decreased (mean variation from baseline $=-1.2$ ). The IADL and ADL scores progressively and mildly declined from baseline to the $36^{\text {th }}$ week (ADL, mean variation from baseline $=$ -0.5 for naïves, -0.3 for non-naïves; $I A D L=-0.7$ for naïves, mean variation from baseline $=-0.4$ ). However, when the MMSE, ADL and IADL variations were controlled for age, sex and education, no significant time effect was found (MMSE, Wilks' lambda $\mathrm{p}=0.34$; $A D L$, Wilks' lambda $\mathrm{p}=0.25$; IADL, Wilks' lambda $\mathrm{p}=0.3$, respectively). These patterns were apparently unrelated to ChE-I doses. Neuroleptic use doubled in naïves and antidepressants increased in both groups. Conclusions: This multi-level therapeutic approach seems to slow down progression in cognitive and functional performance, in both naïve and non-naïve subjects. The possibility of recurrent examinations by specialized physicians, accurate, close management of psychotropic drugs, and informal counseling to caregivers probably aid in achieving such results in a "real world" population of $A D$ elderly subjects living at home. Future studies are needed to assess whether a multi-level therapeutic approach including higher ChE-I dose may perform better in these subjects.

(Aging Clin Exp Res 2005; 17: 54-61)

@2005, Editrice Kurtis

\section{INTRODUCTION}

Recently, the Italian Ministry of Health started a twoyear (September 2000-September 2002) national project

Key words: Alzheimer's disease, caregiver's education, cholinesterase inhibitors, multidimensional approach, psychotropic drugs, "real world". Correspondence: G. Bellelli, MD, Rehabilitation Unit, Ancelle della Carità Hospital, 26100 Cremona, Italy.

E-mail: giuseppebellelli@libero.it

Received February 17, 2004; accepted in revised form October 14, 2004. 
("CRONOS project", www.Alzheimer-cronos.org) for Alzheimer's disease (AD) subjects living in free-dwelling communities. The project was the first on a national level and unique in Europe. It had two main objectives: increasing public awareness about the problem of dementia and related disorders, and an innovative therapeutic approach to $\mathrm{AD}$ subjects and caregivers. Five hundred and three dedicated centers, called Alzheimer Evaluation Units (AEUs), were created throughout Italy, 71 of these located in the Lombardy Region (8 million inhabitants). In these AEUs, specialized teams including geriatricians, neurologists, psychiatrists and psychologists undertook clinical and multi-dimensional assessment of subjects with suspected cognitive impairment. The project was preceded by a six-month advertising campaign on radio, TV, internet and newspapers; in addition, the Ministry of Health mailed to all Italian family practitioners a letter illustrating the aims of the project, procedures for access, and the nature of services. Access to the AEUs was via the National Health system (free-of-charge for AD subjects) after specific requests from family practitioners. The services provided by each $\mathrm{AEU}$ included drugs (free provision of ChE-I drugs, prescriptions for antidepressants and of neuroleptics for behavioral and psychotic symptoms of dementia) and non-pharmacologic approaches (periodic multi-dimensional assessment, informal caregiver counseling on management of dementia and drug-related problems).

In this paper we present the results of this innovative multi-level therapeutic approach after 36 weeks of survey in a population of free-dwelling community $\mathrm{AD}$ subjects enrolled in the CRONOS project. The first aim of this report is to describe - in a setting most closely resembling the everyday clinical practice ("real-world practice") subjects' changes in cognitive performance related to this model of care. The second aim of the study is to examine some specific components of this multi-level approach, including the use of psychotropic and antidepressant drugs.

\section{MATERIALS AND METHODS}

Inclusion criteria for the CRONOS project

Subjects presenting at the AEUs were required to be accompanied by a caregiver and to meet the following criteria:

1. diagnosis of probable $\mathrm{AD}$, according to the criteria of the Diagnostic and Statistical Manual of Mental Disorders, fourth edition (DSM-IV) (1) and National Institute of Neurologic and Communicative Disorders and Stroke-AD and Related Disorders Association (NINCDS-ADRDA) for possible or probable AD (2);

2. Mini Mental State Examination (MMSE) (3) at baseline ranging from 11 to 26 ;

3. onset of cognitive disorders between 40 and 90 years of age.
The occurrence of one of the following conditions: death, nursing home placement, discontinuation of therapy, or MMSE score lower than or equal to 10, caused the removal of the subject from the CRONOS project.

Description of activity of Eastern Lombardy AEUs and of multi-level therapeutic approach

During the study period, more than 60,000 subjects were screened throughout Italy. Of these, about $60 \%$ met the eligibility criteria for CRONOS enrolment on a national level and about one-fifteenth in the Lombardy Region. Three coordination areas were set up in this Region, one of which was located in Eastern Lombardy. At the beginning of the CRONOS project, all 31 AEUs of Eastern Lombardy were contacted and 14 accepted participation in our study, sharing all data in a common database.

Prior to the creation of the common database, the teams of the $14 \mathrm{AEUs}$ had three introductory meetings to standardize the diagnostic procedures for dementia (i.e., similar clinical examinations, hematological analysis, neuropsychological tests, examination of CT scans or MR images of the brain, and 12-lead electrocardiographs) and thus to harmonize the therapeutic approach. For subjects never previously treated with ChE-I drugs (naïve subjects), all physicians agreed to start treatment at the lowest dose $(5 \mathrm{mg}$ daily for donepezil, $6 \mathrm{mg}$ daily for rivastigmine, $8 \mathrm{mg}$ daily for galantamine) to titrate the dosages slowly and to reach the maximum tolerated regimen within approximately 3 months. For subjects already treated with ChE-Is before the start of CRONOS project (non-naiive subjects), physicians agreed to maintain (preferably) the current drug and dose, with the possibility of making pharmacologic adjustments according to their clinical judgement. For both naïve and non-naive subjects, it was decided to use neuroleptics and antidepressants in conformity with the criterion of the minimum effective dose, also leaving in this case the possibility of prescribing other new psychoactive substances or reducing unnecessary drugs. Furthermore, all physicians agreed to spend some time at the end of each visit with caregivers, in order to give them educational support regarding the problems related to drug administration and dementia care. Caregivers were also allowed to contact physicians whenever they wished and, if necessary, to request an extra appointment.

\section{Clinical and multi-dimensional assessment}

Each physician's evaluation started with an interview with the caregiver and included clinical and multi-dimensional assessments. Standard clinic laboratory assessments were carried out only at baseline and included hematological analysis (hemoglobin, hematocrit, total erythrocytes, erythrocyte indices, total and differential leukocyte counts, platelet count), clinical chemistry analysis (including assessments of hepatic, metabolic and re- 
nal functions), and urine analysis ( $\mathrm{pH}$, glucose, protein, hemoglobin or blood, ketosis, specific gravity, microscopic examination of sediment). At the end of each examination, physicians provided caregivers with the exact quantity of ChE-I drug until the next follow-up. Follow-ups were set at 12 and 36 weeks after baseline. The data recorded were:

- demographic characteristics: age, gender, years of education, at baseline; duration of disease was determined, if possible, by clinical records and proxy referral; - cognitive status: administration of the MMSE at baseline and follow-ups;

- functional status: proxy evaluation of independence in Activities of Daily Living (ADL) and Instrumental Activities of Daily Living (IADL), at baseline and follow-ups $(4,5)$;

- somatic health status: revision of clinical records, if any, and proxy referral about the presence of chronic diseases potentially contraindicating ChE-I treatment (i.e. diabetes, hypertension, asthma, arrhythmia, chronic obstructive pulmonary disease);

- previous or current use of medications acting on the central nervous system (CNS) (i.e., ChE-I, neuroleptics, antidepressants, anticonvulsants, long- or short-acting benzodiazepines) at baseline and at the 36-week follow-up; - adverse effects were recorded at each clinical examination and a possible causative relationship with ChE-I drugs was determined.

If patients missed a follow-up, they were contacted by phone and requested to show up within seven days; if unavailable, they were considered as drop-outs.
Informed consent was obtained by AD subjects, if possible, or by their legal representatives. The study was approved by the Ethics Committee of Gerontological Sciences of the Geriatric Research Group, Italy.

\section{Statistical analysis}

Descriptive statistics are presented as mean values and standard deviations or percentages, according to the nature of the variable. Comparisons of the mean values between groups were evaluated by the General Linear Model (GLM); homoschedasticity was first checked by Levene's test, and Welch's test was used when the null hypothesis of homoschedasticity was rejected. Comparisons of MMSE, IADL and ADL mean within-group values were made using the repeated-measure ANOVA by means of the GLM procedure and the repeated option, controlling for age, sex and education. Since the sphericity tests failed for all three measures, only MANOVA results are interpreted.

The association between categorical variables and groups was evaluated by the chi-square test or Fisher's test when the former was not applicable.

All analyses were performed with the 8.02 SAS package.

\section{RESULTS}

Study attrition is shown in Figure 1. A total of 1362 subjects were examined at least once in the 14 AEUs from September 1, 2000 to December 31, 2002. Of these, 338 subjects $(24.7 \%)$ had not completed at this date the 36-week follow-up survey, while 808 (59.3\%) did complete all follow-ups. Eighty-two subjects discontin-

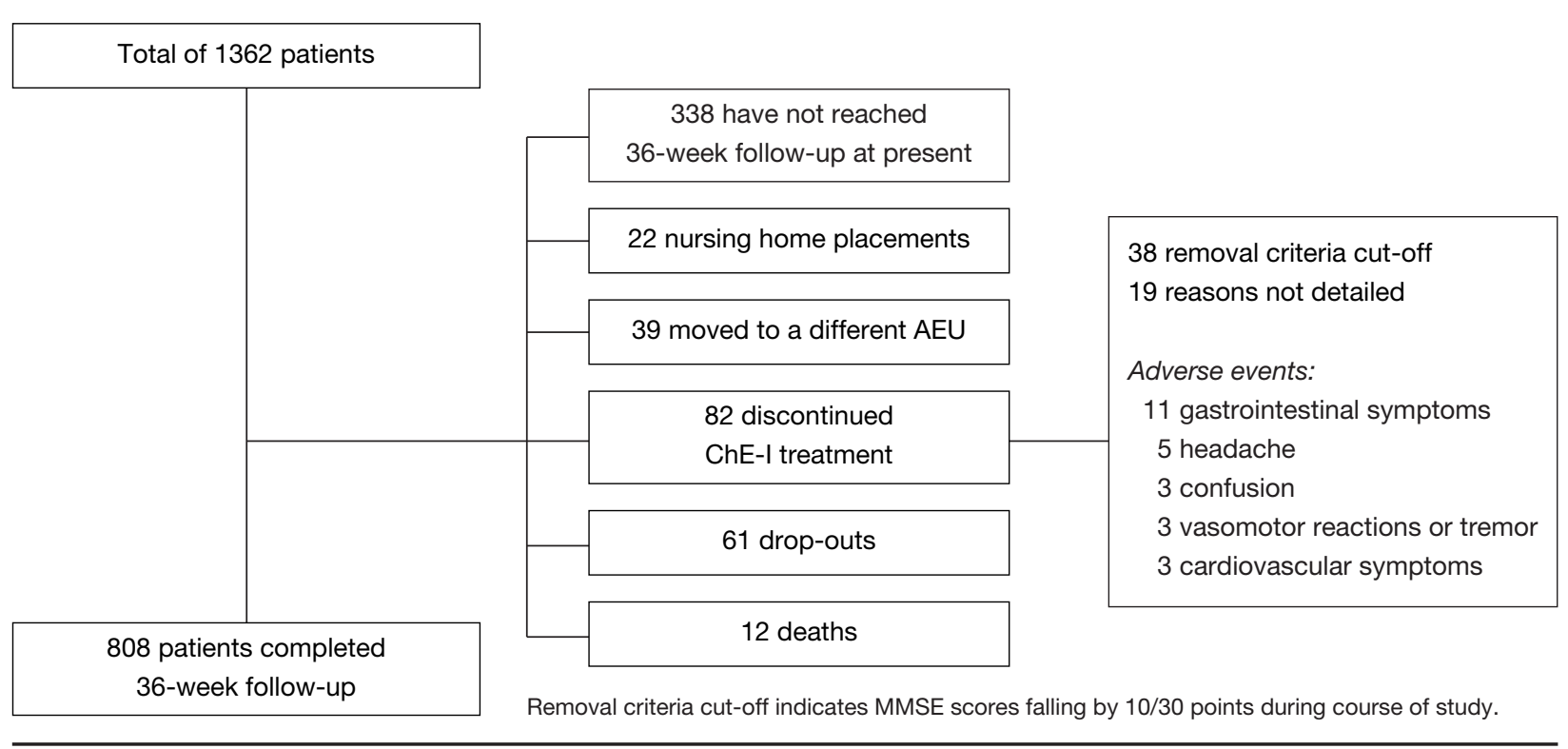

Figure 1 - Study attrition. 
ued ChE-I treatment because of adverse events, 61 dropped out (causes unknown), 39 moved to a different AEU, 22 entered nursing homes, and 12 died. No differences were found at baseline between these groups, reciprocally (data not shown).

Table 1 shows the main characteristics of the 808 subjects who completed the 36-week follow-up, in general and stratified into naïves and non-naïves. On the whole, subjects were old and predominantly women, with more than 2 years mean duration of dementia, and mild to moderate baseline impairment in both cognitive and functional status. About $60 \%$ had at least one or more chronic conditions potentially contraindicating treatment with ChE-I. At the $12^{\text {th }}$ week, their MMSE scores improved (19.2 at baseline us 19.8; paired $t$-test $p$-value $\leq 0.005$ ) but declined at the $36^{\text {th }}$ week (19.2 at baseline us 18.9; paired $t$-test $p$-value $\leq 0.005$ ); the ADL and IADL scores mildly and progressively declined from baseline to

Table 1 - Demographic, cognitive, functional and somatic characteristics in 808 patients completing 36-week follow-up, according to time of initiation of ChE-I treatment.

\begin{tabular}{|c|c|c|c|c|}
\hline & Total & $\begin{array}{c}\text { Naiive } \\
(n=441)\end{array}$ & $\begin{array}{c}\text { Non-naive } \\
(n=367)\end{array}$ & $p$-value* \\
\hline Age & $77.2 \pm 6.7$ & $77.6 \pm 6.2$ & $76.7 \pm 7.3$ & 0.077 \\
\hline Sex (\% female) & 73.9 & 74.1 & 73.6 & NS \\
\hline Education (yrs) & $5.4 \pm 2.6$ & $5.4 \pm 2.7$ & $5.4 \pm 2.6$ & NS \\
\hline Duration of disease (months) & $28.2 \pm 16.0$ & $23.8 \pm 12.1$ & $32.2 \pm 17.9$ & 0.000 \\
\hline $\begin{array}{l}\text { Concurrent chronic diseases (\%) } \\
\text { None } \\
1 \text { or } 2 \\
\geq 3\end{array}$ & $\begin{array}{c}41.8 \\
54.8 \\
3.3\end{array}$ & $\begin{array}{c}40.8 \\
55.8 \\
3.4\end{array}$ & $\begin{array}{c}43.1 \\
53.7 \\
3.3\end{array}$ & NS \\
\hline MMSE score at baseline & $19.2 \pm 3.8$ & $19.6 \pm 3.6$ & $18.7 \pm 4.0$ & 0.001 \\
\hline MMSE score at 12 weeks & $19.8 \pm 4.4$ & $20.4 \pm 4.1$ & $19.2 \pm 4.7$ & 0.000 \\
\hline MMSE score at 36 weeks & $18.9 \pm 4.9$ & $19.7 \pm 4.5$ & $18.0 \pm 5.1$ & 0.000 \\
\hline ADL (functions preserved) at baseline & $4.9 \pm 1.5$ & $5.0 \pm 1.5$ & $4.8 \pm 1.5$ & NS \\
\hline ADL (functions preserved) at 12 weeks & $4.8 \pm 1.5$ & $5.0 \pm 1.4$ & $4.7 \pm 1.6$ & 0.01 \\
\hline ADL (functions preserved) at 36 weeks & $4.5 \pm 1.7$ & $4.5 \pm 1.7$ & $4.5 \pm 1.7$ & NS \\
\hline IADL (functions preserved) at baseline & $3.8 \pm 2.6$ & $4.0 \pm 2.7$ & $3.5 \pm 2.5$ & 0.008 \\
\hline IADL (functions preserved) at 12 weeks & $3.7 \pm 2.6$ & $3.9 \pm 2.6$ & $3.5 \pm 2.5$ & 0.015 \\
\hline IADL (functions preserved) at 36 weeks & $3.2 \pm 2.5$ & $3.3 \pm 2.6$ & $3.1 \pm 2.5$ & NS \\
\hline Previous ChE-I (\%) & 45.4 & 0 & 100 & \\
\hline Mean time of ChE-I assumption (months) & -- & -- & $17.4+12.5$ & \\
\hline $\begin{array}{l}\text { ChE-I drugs (\% at baseline) } \\
\text { Donepezil } \\
\text { Rivastigmine\# } \\
\text { Galantamine\# }\end{array}$ & -- & $\begin{array}{c}68.7 \\
26.1 \\
5.2\end{array}$ & $\begin{array}{l}75.2 \\
19.3 \\
5.4\end{array}$ & NS \\
\hline $\begin{array}{l}\text { ChE-I dose at baseline (mg) } \\
\text { Donepezil } \\
\text { Rivastigmine\# } \\
\text { Galantamine\# }\end{array}$ & -- & $\begin{array}{l}5.0 \pm 0.5 \\
2.9 \pm 0.6 \\
7.8 \pm 0.8\end{array}$ & $\begin{array}{l}6.6 \pm 2.3 \\
4.0 \pm 2.1 \\
8.5 \pm 3.6\end{array}$ & $\begin{array}{l}0.000 \\
0.000 \\
\text { NS }\end{array}$ \\
\hline $\begin{array}{l}\text { ChE-I drugs (\% at } 36 \text { weeks) } \\
\text { Donepezil } \\
\text { Rivastigmine\# } \\
\text { Galantamine\# }\end{array}$ & -- & $\begin{array}{c}67.1 \\
26.1 \\
6.8\end{array}$ & $\begin{array}{l}75.5 \\
18.8 \\
5.7\end{array}$ & 0.030 \\
\hline $\begin{array}{l}\text { ChE-I dose at } 36 \text { weeks (mg) } \\
\text { Donepezil } \\
\text { Rivastigmine }{ }^{\#} \\
\text { Galantamine }^{\#}\end{array}$ & -- & $\begin{array}{c}8.3 \pm 2.4 \\
6.7 \pm 2.2 \\
14.5 \pm 4.8\end{array}$ & $\begin{array}{c}8.5 \pm 2.4 \\
6.4 \pm 2.6 \\
13.5 \pm 6.9\end{array}$ & $\begin{array}{l}\text { NS } \\
\text { NS } \\
\text { NS }\end{array}$ \\
\hline Antidepressants (\% at baseline) & 17.5 & 17.5 & 17.4 & NS \\
\hline Neuroleptics (\% at baseline) & 6.3 & 4.5 & 8.5 & 0.023 \\
\hline Antidepressants (\% at 36 weeks) & 19.6 & 19.7 & 19.5 & NS \\
\hline Neuroleptics (\% at 36 weeks) & 8.4 & 8.8 & 8.0 & NS \\
\hline
\end{tabular}


the $36^{\text {th }}$ week. Three-quarters of all subjects $(n=612$, 77\%) experienced at least one adverse clinical event, including symptoms which were either CNS-related (i.e., agitation, insomnia, confusion, depression, anxiety, dizziness) or gastroenteric system-related (i.e. diarrhea, nausea, abdominal disturbance, stomach upset, anorexia, bloating), but not leading to discontinuation of treatment (data not shown).

Comparing naïve us non-naïve groups, the latter had obviously a longer duration of disease and were more impaired at baseline in both cognitive and IADL (but not $\mathrm{ADL}$ ) functional status. As expected, doses of donepezil, rivastigmine and galantamine were significantly higher in non-naïves at baseline, but tended to overlap at 36 weeks. About one-sixth of all subjects in the two groups took antidepressants at baseline and about one-fifth at 36 weeks; differently, the use of neuroleptics nearly doubled in naives from baseline to 36 weeks, but remained stable in non-naives during the course of the survey. With regard to MMSE values, naïves scored significantly better in comparison with non-naïves at baseline $(p=0.001)$, at 12 weeks $(p<0.001)$ and at 36 weeks $(p<0.001)$; after controlling for age, sex and education, the repeated-measure ANOVA found no significant time effect (Wilks' lambda $p=0.34$ ) and a significant interaction time with groups $(p=0.06)$. The pattern of decline in ADL was different from baseline to 36 weeks between groups (interaction time with group $p=0.014$ ) but it was similar for IADLs (interaction time with group $p=0.08$ ). For both functional measures, no time effect was detected, after controlling for age, sex and education (ADL, Wilks' lambda $p=0.25$; IADL, Wilks' lambda $p=0.3$, respectively).

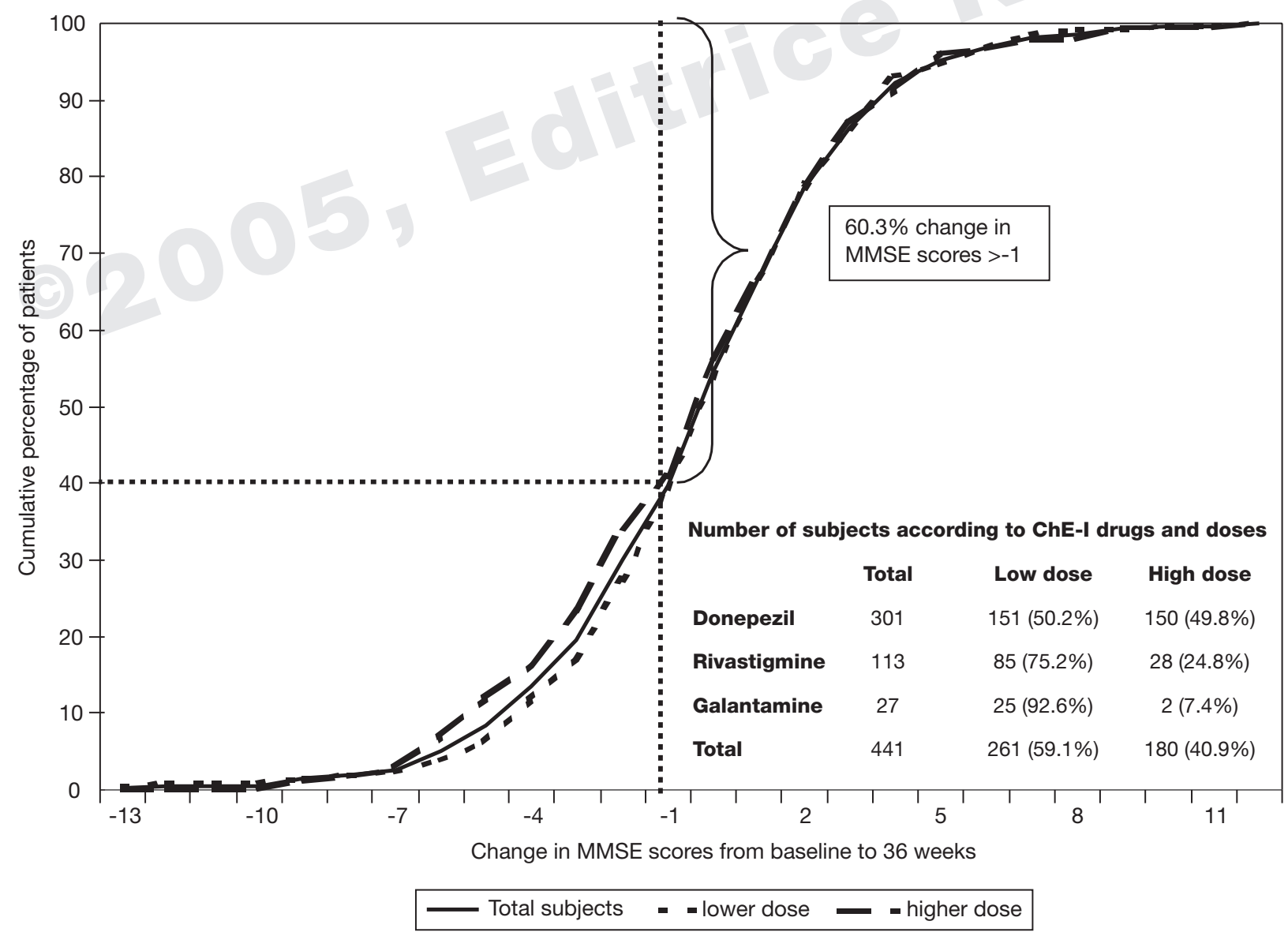

Solid line: total population.

Dotted line: subjects whose ChE-I doses at 36 weeks were less than or equal to $5.0 \mathrm{mg} /$ day for donepezil, $6.0 \mathrm{mg} / \mathrm{day}$ for rivastigmine, and $16.0 \mathrm{mg} /$ day for galantamine; dashed line: subjects whose ChE-I doses at 36 weeks were higher than above-mentioned figures.

Figure 2 - Cumulative percentage of 441 naïve patients for selected variations in MMSE scores from baseline to 36 weeks, according to ChE-I dosage. 
Figure 2 shows the cumulative percentage distribution of the 441 naive subjects according to their variations in MMSE score from baseline to 36 weeks (solid line); the dotted line represents subjects whose ChE-I doses at 36 weeks were less than or equal to $5.0 \mathrm{mg} /$ day for donepezil, $6.0 \mathrm{mg} /$ day for rivastigmine, and $16.0 \mathrm{mg} /$ day for galantamine; the dashed line represents subjects who received higher doses than the above-mentioned ones during the course of the survey. Two hundred and sixty-six subjects (60.3\%) had MMSE variations greater than or equal to zero and about $15 \%$ gained three or more MMSE points during the course of the survey. Instead, $26.3 \%$ lost 1 to 3 points and only $13.4 \%$ more than 4 points at 36 weeks. Interestingly, a significant proportion (59.2\%) of all subjects did not increase drug regimens during the period of survey and, in particular, about half of those assigned to donepezil shifted to higher doses, but only a quarter (24.7\%) of those on rivastigmine and less than $8 \%$ of those on galantamine. It was also observed that the proportion of subjects increasing doses (dashed line, bottom left in Figure) was greatest among those with worse outcomes, indicating that physicians tended to increase drug doses only when no cognitive improvement was observed at the first follow-up.

\section{DISCUSSION}

Several therapeutic approaches have been proposed to stabilize or improve cognition and functional status in $\mathrm{AD}$ subjects. In the past years, many randomized clinical trials (RCTs) have consistently reported the benefit of ChE-I drugs, indicating the mean drug effect over placebo as an improvement in cognition roughly equivalent to stemming 6-12 months of natural decline in untreated subjects (6-13). The correct use of both antipsychotic drugs and antidepressants in behavioral disorders has also been suggested to enhance patients' quality of life, to stabilize cognitive and functional performance, and to reduce caregivers' burden and institutionalization (14-16). Many other studies have also demonstrated that geriatric assessment $(17,18)$ and training of caregivers $(19,20)$ are associated with more favorable outcomes in mild dementia.

The first of its kind in Italy, the CRONOS project attempted to include all these strategies in a unique multicomprehensive approach for mild-to-moderate AD subjects living in the community. The approach included a 2year free-of-charge provision of ChE-I, pharmacologic and non-pharmacologic management of psychotic and depressive disorders, periodic multi-dimensional assessment of clinical, cognitive and functional status, and informal counseling to caregivers on the management of drug- and dementia-related problems. The present study was carried out in the Eastern Lombardy Region as a part of the CRONOS project. Our main aim was to assess the results of this multi-level approach in a setting most resembling everyday clinical practice ("real-world prac- tice"), measuring subjects' variations in cognitive and functional performance at 9 months. Our secondary aim was to assess the prescribing patterns of psychotropic drugs (including ChE-Is) by AEU physicians in the same population.

The main result of the study was the lack of progression in cognitive and functional decline at 9 months, in both naïve and non-naïve subjects. Although the crude scores indicate an improvement in MMSE scores (which ameliorated at 12 weeks and returned to baseline levels at 36 weeks), with a mild and progressive decline in IADL and ADL scores (decreasing from baseline to 36 weeks), when these data were adjusted for sex, age and gender no time effect was found, suggesting that their mean values do not change over time in the two groups. These patterns were apparently unrelated to ChE-I dosages in naïves, as shown by the cumulative percentages for selected MMSE variations (Fig. 2), and also in non-naïves, since most of them took greater ChE-I dosages at baseline compared with naives. Use of neuroleptics doubled in naïves from baseline to 36 weeks, and antidepressants increased in both groups, although not significantly.

Taken together, these results are consistent with the positive effect of the multi-level therapeutic approach. The patterns of MMSE variation observed at 9 months in the global population, and especially in naive subjects, is clearly above the expected rate of natural decline in untreated patients $(21,22)$ and similar to the rate of RCTs (13). In non-naïves, who were expected to decline at a faster rate than naïves because of longer ChE-I drug receptor stimulation, the finding of an improvement in MMSE scores at 12 weeks and of a lack of cognitive decline at 9 months may be viewed as non-progression of the disease, and is comparable with the results of previous open-label extension trials $(10,11)$. Furthermore, the fact that these results occurred independently of ChE-I dosages but, conversely, that neuroleptic dosages increased in naïves and antidepressants in both groups, indirectly suggests that the benefits were due mainly to the management of behavioral and affective disorders rather than to any mnemonic improvement. The possibility of recurrent examinations by specialized physicians, accurate and close management of psychotropic drugs, and informal counseling to caregivers probably represented the clue to these results in our population. Additionally, these data suggest that treatment of dementia-related problems and the ability to deal with caregivers' needs should be an important target for physicians and that the multi-level therapeutic approach should represent the ideal model of care.

Our study is unique in that it was performed in the "real world", i.e., in an environment reflecting as much as possible what routinely occurs in clinical practice. It is known that individuals participating in RCTs result from rigorous selection criteria and often tend to be younger, 
better educated, wealthier, and less behaviorally disturbed (23-27) than subjects not enrolled in trials. Furthermore, care for subjects in clinical trials is delivered in circumstances that differ substantially from those in community clinics (23). In this perspective, RCT participants are representative of only a minority of community $A D$ populations, and the findings are unlikely to be directly translated into physicians' clinical practice $(22,25,26,28)$. Instead, enrolment in the CRONOS project was not prevented by explicit exclusion criteria, but had to satisfy only three simple requirements - i.e., $\mathrm{AD}$ diagnosis, MMSE ranging between 10 to 26/30, and onset of dementia between 40 and 90 years of age. Moreover, AEU physicians were not obliged to follow pre-established therapeutic options, but were free to deliver medical care in a personal manner, on the basis of both their clinical expertise and their ability to interact with patients' problems and caregivers' expectations. As a result, our study contained an $\mathrm{AD}$ population that is commonly not represented in clinical trials (subjects were about five years older and had a longer duration of disease than those of RCTs) $(9,13)$ and physicians' behavior was observed in a less stereotyped clinical condition.

At least three limitations of this study should be highlighted. First, we acknowledge that there was no comparison placebo group, a fact which may have prevented us from drawing definite conclusions about the efficacy of this multi-level approach. However, since this was a national project, it was neither possible nor ethical to exclude AD subjects who had previously been following one or more therapeutic approaches, the benefits of which are well recognized. Furthermore, it seemed inappropriate to use subjects excluded from the CRONOS project as a comparison group because they did not meet the NINCDS-ADRDA criteria for AD. Second, we used only one, relatively gross, measure of cognitive performance, i.e., the MMSE. However, also in this case it should be emphasized that the CRONOS project did not consider instruments other than those of the established protocol; in addition, the adoption of more refined cognitive instruments is commonly very time-consuming and requires a certain degree of familiarity with the administration procedures, clearly contradicting the philosophy of the "real world". In this view, the MMSE, which is easy and quick to administer, represents the ideal tool to assess cognition reliably in clinical practice (16). Third, we are not able to assess whether those who dropped out, moved to different AEUs, or entered nursing homes experienced any serious or minor adverse events; however, it is of interest that the baseline clinical characteristics were not different between these subjects and those who completed the 36-week survey, indirectly suggesting that the causes of treatment withdrawal were not related to clinical condition.
We do not know whether higher-dose ChE-I treatment would perform better in a similar "real world" population. Future studies are needed to answer this question, and also to evaluate if the multi-level therapeutic approach described here could improve quality of life and reduce institutionalization.

\section{APPENDIX}

The following are members of the ELCoA (Eastern Lombardy Coordination Area) study group:

Giuseppe Bellelli, Elena Lucchi, Francesca Magnifico, Alessandra Marrè Brunenghi, Fabio Guerini, "Ancelle della Carità" Hospital, Cremona; Attilio Calza, Sara Mondini, Roberta Baronio, "Figlie di S. Camillo" Hospital, Cremona; Daniele Villani, M.Chiara Ubezio, "Istituto Ospedaliero di Sospiro", Sospiro (Cremona); Stefano Avanzi, Emanuela Galante, IRCCS "Fondazione Maugeri", Castelgoffredo (Mantova); Tommaso Riccardi, Luciana Groppelli, Department of Neurology, Crema Hospital, (Cremona); Alessandro Padovani, Luca Rozzini, Barbara Vicini Chilovi, Department of Neurology, University of Brescia; Angelo Bianchetti, Giovanna Cipriani, "S. Anna" Hospital, Brescia; Renzo Rozzini, Stefano Boffelli, "Poliambulanza" Hospital, Brescia; Simone Franzoni, CMR Richiedei, Palazzolo sull'Oglio (Brescia); Marinella Turla, Department of Neurology, "Valle Camonica" Hospital, Esine (Brescia); Renzo Polotti, Alberto Ranzenigo, Bruno Bertozzi, "Fatebenefratelli" Hospital, Brescia; Francesco Saviotti, Neurology Hospital, Leno (Brescia); Francesco Chia, Department of Neurology, Chiari Hospital, Brescia; Cesare Bargnani, Neurology Unit, "S. Rocco in Franciacorta" Hospital, Ome, Brescia.

\section{ACKNOWLEDGEMENT}

This study was supported by grants of "Progetto Finalizzato Regione Lombardia- Ministero della Salute" to the Geriatric Research Group.

\section{REFERENCES}

1. American Psychiatric Association. Diagnostic and Statistical Manual of Mental Disorders, 4th ed. Washington, D.C.: American Psychiatric Association, 1994.

2. McKhann G, Drachman D, Folstein M, Katzman R, Price D, Stadlan EM. Clinical diagnosis of Alzheimer's disease: report of the NINCDS-ADRDA Work Group under the auspices of Department of Health and Human Services Task Force on Alzheimer's Disease. Neurology 1984; 34: 939-44.

3. Folstein MF, Folstein SE, McHugh PR. "Mini-Mental State". A practical method for grading the cognitive state of patients for the clinician. J Psychiatr Res 1975; 12: 189-98.

4. Katz S, Downs TD, Cash HR, Grotz RC. Progress in development of the index of ADL. Gerontologist 1970; 10: 20-30.

5. Lawton MP, Brody EM. Assessment of older people: self-maintaining and instrumental activities of daily living. Gerontologist 1969; 9: 179-86.

6. O'Brien JT, Ballard CG. Drugs for Alzheimer's disease. BMJ 2001; 323: 123-4.

7. Rogers SL, Farlow MR, Doody RS, Mohs R, Friedhoff LT. A 24week, double-blind, placebo-controlled trial of donepezil in patients with Alzheimer's disease. Donepezil Study Group. Neurology 1998; 50: 136-45.

8. Corey-Bloom J, Anand R, Veach J. A randomized trial evaluating the efficacy and safety of ENA 713 (rivastigmine tartrate), a new acetylcholinesterase inhibitor, in patients with mild to moderately severe Alzheimer's disease for the ENA 713 B352 Study Group. Int J Geriatr Psychopharmacol 1998; 1: 55-65. 
9. Wilcock GK, Lilienfeld S, Gaens E. Efficacy and safety of galantamine in patients with mild to moderate Alzheimer's disease: multicentre randomized controlled trial. Galantamine International-1 Study Group. BMJ 2000; 321: 1445-9.

10. Rogers SL, Doody RS, Pratt RD, leni JR. Long-term efficacy and safety of donepezil in the treatment of Alzheimer's disease: final analysis of a US multicentre open-label study. Eur Neuropsychopharmacol 2000; 10:195-203.

11. Raskind MA, Peskind ER, Wessel T, Yuan W. Galantamine in AD: a 6-month randomized, placebo-controlled trial with a 6-month extension. The Galantamine USA-1 Study Group. Neurology 2000; 54: 2261-8.

12. Farlow M, Anand R, Messina J Jr, Hartman R, Veach J. A 52week study of the efficacy of rivastigmine in patients with mild to moderately severe Alzheimer's disease. Eur Neurol 2000; 44: 236-41.

13. Winblad B, Engedal K, Soininen H, et al., Donepezil Nordic Study Group. A 1-year, randomized, placebo-controlled study of donepezil in patients with mild to moderate AD. Neurology 2001; 57: 489-95.

14. Trinh NH, Hoblyn J, Mohanty S. Efficacy of cholinesterase inhibitors in the treatment of neuropsychiatric symptoms and functional impairment in Alzheimer Disease. A meta-analysis. JAMA 2003; 289: 210-6.

15. Magni E, Binetti G, Bianchetti A, Trabucchi M. Risk of mortality and institutionalization in demented patients with delusions. $J$ Geriatr Psychiatry Neurol 1996; 3: 123-6.

16. Rainer MK, Masching AJ, Ertl MG, Kraxberger E, Haushofer M. Effect of risperidone on behavioral and psychological symptoms and cognitive function in dementia. J Clin Psychiatry 2001; 62: 894-900.

17. Sands LP, Yaffe K, Covinsky K, et al. Cognitive screening predicts magnitude of functional recovery from admission to discharge in hospitalized elders. J Gerontol 2003; 58: 37-45.
18. Bianchetti A, Frisoni GB, Trabucchi M. Do old age psychiatrists miss physical illness? Int $J$ Geriatr Psychiatry 1993; 8: 356-7.

19. Sabir M, Pillemer K, Suitor J, Patterson M. Predictors of successful relationships in a peer support program for Alzheimer's caregivers. Am J Alzheimers Dis Other Demen 2003; 18: 115-22.

20. Volicer L, Hurley AC. Management of behavioral symptoms in progressive degenerative dementias. J Gerontol 2003; 58: M837-45.

21. Doody RS, Stevens JC, Beck C, et al. Practice parameter: management of dementia (an evidence-based review). Report of the Quality Standards Subcommittee of the American Academy of Neurology. Neurology 2001; 56: 1154-66.

22. Bianchetti A, Padovani A, Trabucchi M. Outcomes of Alzheimer's disease treatment: the Italian CRONOS project. Int J Geriatr Psychiatry 2003; 18: 87-8.

23. Cummings JL. Use of cholinesterase inhibitors in clinical practice: evidence based recommendations. Am J Geriatr Psychiatry 2003; 11: 131-45.

24. Tunis, SR, Stryer DB, Clancy CM. Practical clinical trials. Increasing the value of clinical research for decision making in clinical and healthy policy. JAMA 2003; 290: 1624-32.

25. Frisoni GB. Treatment of Alzheimer's disease with acetylcholinesterase inhibitors: bridging the gap between evidence and practice. J Neurol 2001; 248: 551-7.

26. Albert SM, Sano M, Marder K, et al. Participation in clinical trials and long-term outcomes in Alzheimer's disease. Neurology 1997; 49: 38-43.

27. Schneider IS, Olin JT, Lyness SA, et al. Eligibility of Alzheimer's disease clinic patients for clinical trials. J Am Geriatr Soc 1997; 43: 923-8.

28. Bayer T. Commentary: Another piece of the Alzheimer's jigsaw. BMJ 1999; 318: 633-40. 